\title{
Are intravenous injections of contrast media really less nephrotoxic than intra-arterial injections?
}

\author{
Ulf Nyman • Torsten Almén • Bo Jacobsson • \\ Peter Aspelin
}

Received: 30 October 2011 /Revised: 8 December 2011 / Accepted: 17 December 2011 /Published online: 4 February 2012

(C) European Society of Radiology 2012

\begin{abstract}
We oppose the opinion that the intra-arterial administration of iodine-based contrast media (CM) appears to pose a greater risk of contrast medium-induced nephropathy (CIN) than intravenous administration since 1) in intra-arterial coronary procedures and most other intra-arterial angiographic examinations, $\mathrm{CM}$ injections are also intravenous relative to the kidneys, 2) there is a lack of comparative trials studying the risk of CIN between intra-arterial and intravenous procedures with matched risk factors and CM doses, 3) a bias selection of patients with fewer risk factors may explain the seemingly lower rate of $\mathrm{CIN}$ after $\mathrm{CT}$ in comparison with coronary interventions, 4) the rate of CIN following intra-arterial coronary procedures may also be exaggerated owing to other causes of acute kidney failure, such as haemodynamic instability and microembolisation, 5) roughly the same gramiodine/GFR ratio $(\approx 1: 1)$ as a limit of relatively safe $\mathrm{CM}$ doses has preliminarily been found for both intravenous CT and
\end{abstract}

\footnotetext{
U. Nyman $(\bowtie)$

Department of Diagnostic Radiology, University of Lund, Lasarettet Trelleborg,

SE-231 85 Trelleborg, Sweden

e-mail: ulf.nyman@skane.se

U. Nyman

e-mail: ulf.nyman@bredband.net

T. Almén

Department of Clinical Sciences/Medical Radiology,

University of Lund, Skåne University Hospital,

SE-205 02 Malmö, Sweden

\section{B. Jacobsson}

Department of Diagnostic Radiology, The Queen Silvia Children's Hospital, University of Gothenburg and the Sahlgrenska Academy, SE-416 85 Göteborg, Sweden

\section{P. Aspelin}

Division of Medical Imaging and Technology, Department of

Clinical Science, Intervention and Technology (CLINTEC),

Karolinska Institute and University Hospital,

SE-14186 Stockholm, Sweden

intra-arterial coronary procedures and 6) the substantially higher injected intravenous $\mathrm{CM}$ dose rate during $\mathrm{CT}$ relative to an intra-arterial coronary procedure might actually pose a higher risk of CIN following CT.

Key Points

- Most intra-arterial injections of contrast media are intravenous relative to the kidneys.

- No evidence that intravenous CM injections should be less nephrotoxic than intra-arterial.

- Considerably higher dose rates of CM are used for CT relative to intra-arterial procedures.

- Higher dose rates may pose higher nephrotoxic risk for intravenous based CT studies.

Keywords Angiography · Acute kidney injury · Computed tomography $\cdot$ Contrast media $\cdot$ Renal insufficiency

\section{Introduction}

Iodine contrast media ( I-CM) may be among the most commonly used medications with an estimated 80 million doses given worldwide in 2003 [1]. In year 2000, an estimated 1,790,000 cardiac catheterisations were performed in the USA resulting in more than $59,000(3 \%)$ cases of contrast medium-induced nephropathy (CIN) of which about $10 \%$ required haemodialysis [2]. Similar percentage figures were recently reported in a US registry study [3].

Consensus panels [4], recent editorials $[5,6]$ and the 2011 updated ESUR Contrast Media Safety Committee guidelines [7] state that intra-arterial (IA) administration of I-CM appears to pose a greater risk for CIN than intravenous (IV) administration based on the following:

1) The reported frequency of CIN following IV administration of $\mathrm{CM}$ has been suggested to be lower than that following cardioangiography and other IA procedures [4-6]. 
2) Controlled studies that support the hypothesis that IV injected CM is potentially nephrotoxic are lacking $[6,8]$ and

3) The serum creatinine ( $\mathrm{sCr}$ ) in hospitalised patients not exposed to $\mathrm{CM}$ increases about as often as it does in published series of patients receiving IV CM; this indicates an overestimation of the risk of CIN [9].

The arguments above have led to suggestions such as:

1. "The apparently lower risk of CIN associated with IV CM administration in clinical settings such as contrast-enhanced multidetector CT makes it defensible to consider using CM even in patients with greater levels of background risk factors (e.g. greater degrees of pre-existing chronic renal insufficiency) than one would be comfortable with in the IA setting..." [5] and

2. "International radiologic professional organisations... should revisit the basis of their practice guidelines to reduce their implications about the danger of CIN with CMenhanced CT" [6].

3. An estimated GFR $<60 \mathrm{~mL} / \mathrm{min} / 1.73 \mathrm{~m}^{2}$ is considered a risk factor for IA administration, while GFR is not considered a CIN risk threshold for IV CM until it falls below 45 $\mathrm{mL} / \mathrm{min} / 1.73 \mathrm{~m}^{2}$ [7].

In the present paper we disagree with the suggestions that IV injections of CM may be less nephrotoxic than IA injections. We fear that these suggestions may jeopardise patient safety by hinting at the use of higher IV CM doses in azotaemic patients without reference to any studies comparing the risk of CIN following IV and IA injections in cohorts with matched CM doses and risk factors. To our knowledge, the only direct comparison of the incidence of CIN between IV and IA administration of CM was published recently [10]. Each patient underwent both CT angiography and digital subtraction angiography (DSA) of the aorto-femoral arteries. Although the study was hampered by lack of power analysis and sCR measurements only at $24 \mathrm{~h}$ postexamination, no significant difference in the rate of CIN was found. The lack of difference occurred despite the fact that the DSA results may have been affected by the CM load from the CT performed 3-14 days before the DSA. Apart from this study our main arguments that IV injections may be as nephrotoxic as IA injections are based on the following.

\section{Intra-arterial injections are mainly intravenous relative to the kidneys}

In view of the general statement that IA administration of $\mathrm{CM}$ is more nephrotoxic than IV injections; does that imply that $\mathrm{CM}$ drained via the arm veins following selective subclavian arteriography should be more nephrotoxic than if the same amount was injected into the antecubital vein in the same patient? Although the opinion about the higher nephrotoxicity of IA injections primarily refers to diagnostic and percutaneous coronary interventions (PCI), the CM molecules still have to pass through the coronary arteries to the right atrium via the coronary veins. To us it seems inexplicable that this should be more nephrotoxic than if the same molecules pass via the arm veins to the right atrium and then through the pulmonary circulation to finally reach the kidneys.

It should be noted that in most IA injections besides coronary and subclavian artery injections, the $\mathrm{CM}$ has to pass the venous system before reaching the kidneys (e.g. carotid, coeliac, mesenteric, distal aortic and iliaco-femoral), i.e. IA injections are frequently IV relative to the kidneys. An exception to this is CM injected for left ventriculography. However, even then only a minor portion will reach the kidneys directly during the first pass through the aorta, i.e. about $20 \%$ of cardiac output or $6-8 \mathrm{~mL}$ of an injected volume of 30 $40 \mathrm{~mL}$. This corresponds to only $2-3 \mathrm{~g}$ of iodine (anticipated concentration $350 \mathrm{mg} \mathrm{I} / \mathrm{mL}$ ) of a total mean dose commonly ranging between 40 and 90 g-I during coronary procedures [11-15]. Thus, if there is a difference in the rate of acute kidney injury (AKI) between IA and IV administration of $\mathrm{CM}$, it may be due to factors (see further discussion below) other than simply the route of administration.

Admittedly, supra- and juxta-renal aortic as well as selective renal injections are true IA CM exposures of the kidneys and should pose a greater risk of CIN than injections that are IV relative to the kidneys. Higher plasma concentrations of CM with a subsequently higher nephrotoxic potential will strike the kidneys, especially if plasma hypertonic solutions are used with possible vascular endothelial injuries and crenation of red blood cells that may affect microcirculation $[16,17]$.

\section{Contrast medium dose and CIN}

One explanation for the perceived difference in CIN rate between $\mathrm{CT}$ and IA cardioangiography may be the lower CM dose used in CT, which commonly ranges between 25 and 50 g-I (Table 1) [18] compared with reported 40 to $90 \mathrm{~g}$-I in IA coronary studies as already mentioned. However, the rate of CIN in CT studies has ranged from $4 \%$ to $28 \%$ (Table 1 ). In one of the studies, an incidence as high as $42 \%$ was observed in a subgroup of patients with marked reduction of renal function ( $\mathrm{sCr}>221 \mu \mathrm{mol} / \mathrm{L}$ ) after receiving $22 \mathrm{~g}$-I from the low osmolality CM (LOCM) iopromide [19]. In two CT studies $[19,20]$ the CIN risk figures are well in line with the $26 \%$ and $33 \% \mathrm{CIN}$ rates of iohexol in patients with renal insufficiency and diabetes mellitus following cardioangiography with a mean dose of about 50 g-I iodine in the Nephric Study [12] and 
Table 1 Literature review of non-randomised and randomised CT studies from 2000 to 2011 reporting mean gram-iodine (g-I) dose (or volume and concentration), mean estimated glomerular filtration rate (eGFR) and incidence of contrast medium-induced nephropathy (CIN; serum creatinine rise $\geq 25 \%$ or $\geq 44 \mu \mathrm{mol} / \mathrm{L}$ above baseline). Based on mean $\mathrm{g}$-I dose and mean eGFR, the $\mathrm{g}-\mathrm{I} / \mathrm{eGFR}$ ratio for each study was calculated. Only results for low-osmolal contrast media (LOCM) were included unless there was no significant difference between LOCM and IOCM (iso-osmolal contrast media) in randomised studies. Weighted mean value of CM dose, eGFR, g-I/eGFR ratio and CIN incidence with individual study sizes as weights were calculated. The weighted mean of the $\mathrm{g}-\mathrm{I} / \mathrm{eGFR}$ ratio was based on log transformation

\begin{tabular}{|c|c|c|c|c|c|c|}
\hline First author, year & Type of CM & $n$ & CM dose $(g-I)$ & eGFR $\left(\mathrm{mL} / \mathrm{min}\right.$ or $\left.\mathrm{mL} / \mathrm{min} / 1.73 \mathrm{~m}^{2}\right)$ & g-I/eGFR ratio & CIN (\%) \\
\hline Tepel $^{\mathrm{a}}, 2000$ [19] & LOCM & 42 & 23 & 34 & 0.7 & 21 \\
\hline Lufft, 2002 [43] & LOCM & 33 & 49 & 63 & 0.8 & 9.1 \\
\hline Kolehmainen, 2003 [44] & LOCM/IOCM & 50 & 35 & 29 & 1.2 & 16 \\
\hline Garcia-Ruiz, 2004 [45] & LOCM & 50 & 48 & 30 & 1.6 & 4.0 \\
\hline Becker, 2005 [46] & LOCM & 100 & 27 & 41 & 0.7 & 9.0 \\
\hline Barrett, 2006 [33] & LOCM/IOCM & 150 & 40 & 45 & $1.0^{\mathrm{b}}$ & 3.9 \\
\hline Thomsen $^{\mathrm{c}}, 2008[35]$ & LOCM/IOCM & 148 & 40 & 42 & 1.0 & 6.1 \\
\hline Nguyen, 2008 [20] & LOCM & 56 & 37 & 53 & 0.7 & 28 \\
\hline Kuhn, 2008 [34] & LOCM/IOCM & 248 & 36 & 49 & 0.7 & 5.2 \\
\hline Weisbord, 2008 [36] & LOCM & 421 & 48 & 53 & 0.9 & 6.5 \\
\hline Kim, 2011 [47] & LOCM & 520 & 40 & 43 & 0.9 & 2.5 \\
\hline Weighted mean data & & 1821 & 40 & 46 & 0.9 & 6.3 \\
\hline
\end{tabular}

${ }^{\text {a }}$ Only control group not receiving acetylcysteine included

${ }^{\mathrm{b}}$ Based on individual data in the report

${ }^{\mathrm{c}}$ Based on the CIN definition $\geq 25 \%$ serum creatinine increase

Iohexol Cooperative study [11], respectively. Thus, the risk of $\mathrm{CIN}$ may be within the same range at similar $\mathrm{CM}$ doses.

\section{Contrast medium dose/GFR ratio as a predictor of CIN}

Contrast medium volume- or grams of iodine-to-estimated glomerular filtration rate (g-I/eGFR) ratio is a way of expressing systemic exposure of a drug cleared by the kidneys and is often well correlated with its toxicity [21, 22]. This ratio has during recent years been studied with the goal of defining a threshold value for the risk of CIN in PCI [13-15, 23-27]. Analysis of these studies demonstrates a weighted mean $\mathrm{g}-\mathrm{I} / \mathrm{eGFR}$ value $>1.2$ to be a significant and independent predictor of CIN (Table 2). A most recently published registry study involving about 50,000 patients showed that the risk of $\mathrm{CIN}$ approached significance when the $\mathrm{CM}$ volume/eGFR ratio exceeded 2 and was dramatically elevated when exceeding a ratio of 3 . These $\mathrm{CM}$ volume/ eGFR ratio thresholds would correspond to a g-I/GFR ratio of 0.7 and 0.9 , respectively, if anticipating a CM concentration of $350 \mathrm{mg} \mathrm{I} / \mathrm{mL}$ for PCI [3].

Reported CIN frequency (sCR rise $\geq 44.2 \mu \mathrm{mol}$ and/or $25 \%$ from baseline) at $\mathrm{g}-\mathrm{I} / \mathrm{eGFR}$ ratios $<1.0$ in the PCI studies were $\leq 3 \%[3,13,14,26,27]$ and mean $\mathrm{g}-\mathrm{I} / \mathrm{GFR}$ ratios in patients without and with CIN ranged between $0.7-$ 1.0 and $1.2-1.8$, respectively $[13,15,23,27]$. Although no such studies based on individual patients exist regarding CM-enhanced CT, pooled analysis of CT studies reporting mean data of g-I dose (or mean volume and concentration) and eGFR demonstrates a weighted mean CIN risk of $6 \%$ at a weighted mean g-I/eGFR ratio of 0.9 (Table 1). This does not support the view that the rate of $\mathrm{CIN}$ in a CT population should be substantially less than that of a PCI population at similar CM dose/GFR ratios.

\section{Severity of CIN and mortality}

It has also been argued that the severity of CIN is less after $\mathrm{CM}$-enhanced $\mathrm{CT}$ with no patients requiring dialysis and no deaths [6]. However, in one controlled study [28] 19\% (14/ 75) of critically ill patients in intensive care units (ICU) undergoing CM-enhanced CT $(140 \mathrm{~mL}$, concentration not stated) without pre-existing renal disease and sCR levels below $133 \mu \mathrm{mol} / \mathrm{L}(<1.5 \mathrm{mg} / \mathrm{dL})$ had a sCR rise $>25 \%$ from baseline. Two of the 14 patients (14\%; $95 \%$ confidence interval 11-29\%) developed CIN requiring haemodialysis. In the matched control group, not receiving $\mathrm{CM}$, only $1 \%$ only $1 \%$ had a $\mathrm{sCr}$ elevation $>25 \%$. In another study on critically ill patients renal replacement therapy was needed within 7 days in $21 \%$ of the patients developing CIN after a CM-enhanced CT [29]. Furthermore, CIN was independently associated with ICU mortality. In a prospective study of unselected emergency patients, all receiving $120 \mathrm{~mL} 370 \mathrm{mg} \mathrm{I} /$ $\mathrm{mL}$ (44 g-I, e-mail correspondence with the authors), 11\% (70/ 633 ) increased their sCR $\geq 44 \mu \mathrm{mol} / \mathrm{L}$ or $\geq 25 \%$ of whom $9 \%$ $(n=6)$ developed severe $\mathrm{CIN}$, which after careful analysis was 
Table 2 Studies on percutaneous coronary interventions defining the threshold of contrast medium volume-to-estimated GFR (eGFR) ratio and corresponding g-I-to-eGFR ratio above which the ratio serves as a significant and independent predictor of contrast medium-induced nephropathy (serum creatinine rise $\geq 25 \%$ or $\geq 44 \mu \mathrm{mol} / \mathrm{L}$ above baseline). Weighted mean value of volume/eGFR and g-I/eGFR ratio with individual study sizes as weights were finally calculated based on logtransformation of volume/eGFR and g-I/eGFR ratio

\begin{tabular}{|c|c|c|c|c|c|}
\hline First author, year & $n$ & Indication & Volume/eGFR ratio & Concentration (mg I/mL) & g-I/eGFR ratio \\
\hline Laskey, 2007 [13] & 3179 & Unselected population & 3.7 & $350^{\mathrm{c}}$ & $1.3^{\mathrm{f}}$ \\
\hline Nyman, 2008 [14] & 391 & STEMI & $2.9^{\mathrm{b}}$ & 350 & 1.0 \\
\hline Nozue, 2009 [25] & 60 & Stable angina & 5.1 & 370 & $1.9^{\mathrm{f}}$ \\
\hline Worasuwannarak, 2010 [15] & 248 & Elective diabetics & 2.6 & $370^{\mathrm{d}}$ & 1.0 \\
\hline Mager, 2011 [24] & 871 & STEMI & 3.7 & 370 & $1.4^{\mathrm{f}}$ \\
\hline Lieu, 2011 [23] & 277 & STEMI & 2.4 & $370^{\mathrm{e}}$ & $0.9^{\mathrm{f}}$ \\
\hline Yoon, 2011 [26] & 226 & Non-emergency & $4.0^{\mathrm{b}}$ & 350 & 1.40 \\
\hline Tan, 2011 [27] & 1140 & Unselected population & 2.6 & $370^{\mathrm{c}}$ & 1.0 \\
\hline Total & $5252^{\mathrm{a}}$ & & & & \\
\hline Weighted mean value & & & 3.5 & & 1.2 \\
\hline
\end{tabular}

STEMI, ST segment elevation myocardial infarction

${ }^{\text {a }} 83 \%$ low-osmolal, $16 \%$ iso-osmolal and $1 \%$ high-osmolal contrast media

${ }^{\mathrm{b}}$ Calculated by the present authors from the $\mathrm{g}-\mathrm{I} / \mathrm{eGFR}$ ratio and iodine concentration

${ }^{\mathrm{c}}$ Mean concentration anticipated by the present authors

d $96 \% 370 \mathrm{mg} \mathrm{I} / \mathrm{mL}$ and $4 \% 320 \mathrm{mg} \mathrm{I} / \mathrm{mL}$ (e-mail communication with the authors)

e 271 patients $370 \mathrm{mg} \mathrm{I} / \mathrm{mL}$ and 6 patients $320 \mathrm{mg} \mathrm{I} / \mathrm{mL}$ (e-mail communication with the authors)

${ }^{\mathrm{f}}$ Calculated by the present authors from the volume/eGFR ratio and iodine concentration

regarded to have contributed to the patient's death in 4 of the 6 cases [30]. Thus, the frequency of patients requiring renal replacement therapy among those developing CIN agrees well with the figures for coronary interventions $[2,3]$.

Another study opposing the opinion that IV injections should be less dangerous than IA injections is the study by From et al [31]. They found that IV CM administration was associated with an increased 30-day and overall mortality compared with IA administration after adjustment for risk factors such as heart failure, CM load, hydration etc. One explanation for this difference might be that IV injections at $\mathrm{CT}$ result in a much higher injected dose rate than IA injections during coronary procedures. A typical $40 \mathrm{~g}$-I dose (100 mL $400 \mathrm{mg} \mathrm{I} / \mathrm{mL}$ ) at CT according to Table 1 injected at $5 \mathrm{~mL} / \mathrm{s}$ results in an injected dose rate of $2.0 \mathrm{~g}-\mathrm{I} / \mathrm{s}$. During an IA coronary procedure multiple small injections of $\mathrm{CM}$ reaching a total dose of $50 \mathrm{~g}-\mathrm{I}[11,12]$ and lasting for $40 \mathrm{~min}$ imply a mean dose rate of only one hundredth of that at CT, i.e. 0.02 $\mathrm{g}-\mathrm{I} / \mathrm{s}$. Thus, at similar $\mathrm{CM}$ doses the much higher injected dose rate during $\mathrm{CT}$ compared with that during PCI may be more toxic to the kidneys. Animal toxicity studies have also shown that higher injection rates of the same $\mathrm{CM}$ dose are accompanied by higher toxicity [32].

\section{Bias selection of patients at risk of CIN}

It should be noted that in both randomised and nonrandomised $\mathrm{CT}$ studies comparing renal toxicity of various
CM, high-risk patients such as those with unstable renal function, heart failure, haemodynamic instability, uncontrolled diabetes, recent $\mathrm{CM}$ examinations etc. are often excluded [20,30,33-36]. This bias in patient selection compared with IA coronary procedures, where high-risk patients cannot be excluded from life-saving interventions, may in part contribute to the belief that IV CM injection implies a lower risk of CIN than IA administration. However, in daily clinical practice $\mathrm{CM}$-enhanced $\mathrm{CT}$ has to be performed despite renal impairment and unstable conditions.

The increased use of coronary CT may in the future also include the type of high-risk patients that so far primarily have undergone IA coronary arteriography. It is therefore of utmost importance not to mislead our colleagues that an IV injection of CM is less dangerous than an IA injection, until proven otherwise using indisputable evidence.

\section{Matched control cohorts and background fluctuation of kidney function}

It has been claimed that controlled studies supporting the hypothesis that IV injected CM is potentially nephrotoxic are lacking $[6,8]$, but as far as we know the same paucity also affects IA coronary studies. It has also been reported that $\mathrm{sCr}$ in hospitalised patients not exposed to $\mathrm{CM}$ may increase as often as it does in published series of patients receiving IV CM indicating an overestimation of the risk of CIN [9]. However, these hospitalised patients may represent 
a selected group of patients at greater risk of AKI and where the physician found reasons to order frequent $\mathrm{sCr}$ measurements. Then sCR may certainly also increase following IA coronary procedures for reasons other than $\mathrm{CIN}$ including haemodynamic instability and microshowers of cholesterol emboli due to catheter manipulations. Thus, it may instead be the case that IA administration of CM may be equally as safe as IV injections. On the other hand it may be argued that $\mathrm{CM}$ may potentiate $\mathrm{AKI}$ in patients hospitalised with conditions leading to increasing $\mathrm{sCr}$ (unstable renal function) such as hemodynamic instability. In another study on background fluctuation of kidney function the incidence of AKI after IV injection of LOCM actually increased above the level of the control cohort when baseline $\mathrm{sCr}$ was $>160 \mu \mathrm{mol} / \mathrm{L}(1.8 \mathrm{mg} / \mathrm{dL})[37]$.

\section{Safety first!}

Based on the present argumentation and lack of convincing evidence we would recommend putting "safety first" and acting accordingly, especially in high-risk patients with an estimated GFR of $<45 \mathrm{~mL} / \mathrm{min}$ [7] or multiple risk factors, congestive heart failure (NYHA class III and IV) or receiving multiple CM exposures within $72 \mathrm{~h}$ irrespective of renal function [38]. In such patients it may be advisable to first consider the pre-test probability of disease and then start with a unenhanced CT whenever possible, scrutinise the examination and supplement with, for example, ultrasound and/or MRI if indicated before embarking on CM-enhanced CT. If CM is deemed necessary the examination should be delayed whenever possible to institute adequate prophylaxis including IV hydration with crystalloids, withdrawal of nephrotoxic drugs and treatment of modifiable risk factors. CM dosing should be performed according to body weight, so at least low-weight patients do not get unnecessarily high doses [39, 40]. CT angiography may be performed with half the ordinary $\mathrm{CM}$ dose or even lower by decreasing the X-ray tube potential from 120 to 80 peak kilovoltage $(\mathrm{kVp})$ [41, 42]. This requires a substantial increase in X-ray tube loading (mAs) so as not to deteriorate image noise and to keep the contrast-to-noise ratio at an acceptable level. The resulting increased radiation dose in these commonly elderly patients at risk of CIN seems be of less concern in this situation. We also have positive experience of performing $80-\mathrm{kVp} \mathrm{CT}$ of the thorax and abdomen with halved CM doses.

\section{Conclusion}

For a number of reasons presented there is no conclusive scientific evidence that IV injections of CM are less nephrotoxic than IA injections. It could also be the case that both routes of administration are equally safe and that AKI after IA coronary procedures may stem from factors other than $\mathrm{CM}$ toxicity. Until we know, recommendations to professional international radiological organisations to dismantle the safety recommendations for $\mathrm{CM}$-enhanced $\mathrm{CT}$ seems premature and unwise until properly performed comparative studies adjusted for risk factors and $\mathrm{CM}$ doses have proven a real difference.

Acknowledgements All authors have received fees from pharmaceutical companies when lecturing about contrast media. T.A. receives royalties for iodixanol from GE Healthcare.

\section{References}

1. Katzberg RW, Haller C (2006) Contrast-induced nephrotoxicity: clinical landscape. Kidney Int Suppl S3-7

2. Gami AS, Garovic VD (2004) Contrast nephropathy after coronary angiography. Mayo Clin Proc 79:211-219

3. Gurm HS, Dixon SR, Smith DE, Share D, LaLonde T, Greenbaum A, Moscucci M (2011) Renal function-based contrast dosing to define safe limits of radiographic contrast media in patients undergoing percutaneous coronary interventions. J Am Coll Cardiol 58:907-914

4. Davidson CJ, Stacul F, McCullough PA, Tumlin J, Adam A, Lameire N, Becker CR (2006) Contrast medium use. Am J Cardiol 98:42K-58K

5. Katzberg RW, Barrett BJ (2007) Risk of iodinated contrast material-induced nephropathy with intravenous administration. Radiology 243:622-628

6. Katzberg RW, Newhouse JH (2010) Intravenous contrast mediuminduced nephrotoxicity: is the medical risk really as great as we have come to believe? Radiology 256:21-28

7. Stacul F, van der Molen AJ, Reimer P, Webb JA, Thomsen HS, Morcos SK, Almen T, Aspelin P, Bellin MF, Clement O, HeinzPeer G (2011) Contrast induced nephropathy: updated ESUR Contrast Media Safety Committee guidelines. Eur Radiol 21:2527-2541

8. Rao QA, Newhouse JH (2006) Risk of nephropathy after intravenous administration of contrast material: a critical literature analysis. Radiology 239:392-397

9. Newhouse JH, Kho D, Rao QA, Starren J (2008) Frequency of serum creatinine changes in the absence of iodinated contrast material: implications for studies of contrast nephrotoxicity. AJR Am J Roentgenol 191:376-382

10. Karlsberg RP, Dohad SY, Sheng R (2011) Contrast mediuminduced acute kidney injury: comparison of intravenous and intraarterial administration of iodinated contrast medium. J Vasc Interv Radiol 22:1159-1165

11. Rudnick MR, Goldfarb S, Wexler L, Ludbrook PA, Murphy MJ, Halpern EF, Hill JA, Winniford M, Cohen MB, VanFossen DB (1995) Nephrotoxicity of ionic and nonionic contrast media in 1196 patients: a randomized trial. The Iohexol Cooperative Study. Kidney Int 47:254-261

12. Aspelin P, Aubry P, Fransson SG, Strasser R, Willenbrock R, Berg KJ (2003) Nephrotoxic effects in high-risk patients undergoing angiography. N Engl J Med 348:491-499

13. Laskey WK, Jenkins C, Selzer F, Marroquin OC, Wilensky RL, Glaser R, Cohen HA, Holmes DR Jr (2007) Volume-to-creatinine clearance ratio: a pharmacokinetically based risk factor for prediction of early creatinine increase after percutaneous coronary intervention. J Am Coll Cardiol 50:584-590

14. Nyman U, Björk J, Aspelin P, Marenzi G (2008) Contrast medium dose-to-GFR ratio: a measure of systemic exposure to predict 
contrast-induced nephropathy after percutaneous coronary intervention. Acta Radiol 49:658-667

15. Worasuwannarak S, Pornratanarangsi S (2010) Prediction of contrast-induced nephropathy in diabetic patients undergoing elective cardiac catheterization or PCI: role of volume-to-creatinine clearance ratio and iodine dose-to-creatinine clearance ratio. J Med Assoc Thai 93(Suppl 1):S29-S34

16. Nyman U, Almen T (1980) Effects of contrast media on aortic endothelium. Experiments in the rat with non-ionic monomeric and monoacidic dimeric contrast media. Acta Radiol Suppl 362:65-71

17. Aspelin P, Stacul F, Thomsen HS, Morcos SK, van der Molen AJ (2006) Effects of iodinated contrast media on blood and endothelium. Eur Radiol 16:1041-1049

18. Johnson PT, Naidich D, Fishman EK (2007) MDCT for suspected pulmonary embolism: multi-institutional survey of 16-MDCT data acquisition protocols. Emerg Radiol 13:243-249

19. Tepel M, van der Giet M, Schwarzfeld C, Laufer U, Liermann D, Zidek W (2000) Prevention of radiographic-contrast-agent-induced reductions in renal function by acetylcysteine. N Engl $\mathrm{J}$ Med 343:180-184

20. Nguyen SA, Suranyi P, Ravenel JG, Randall PK, Romano PB, Strom KA, Costello P, Schoepf UJ (2008) Iso-osmolality versus low-osmolality iodinated contrast medium at intravenous contrastenhanced CT: effect on kidney function. Radiology 248:97-105

21. Chen M-L, Lekso L, Williams R (2001) Measures of exposure versus measures of rate and extent of absorption. Clin Pharmacokinet 40:565-572

22. Sherwin PF, Cambron R, Johnson JA, Pierro JA (2005) Contrast dose-to-creatinine clearance ratio as a potential indicator of risk for radiocontrast-induced nephropathy: correlation of $\mathrm{D} / \mathrm{CrCL}$ with area under the contrast concentration-time curve using iodixanol. Invest Radiol 40:598-603

23. Liu Y, Tan N, Zhou YL, He PC, Luo JF, Chen JY (2012) The contrast medium volume to estimated glomerular filtration rate ratio as a predictor of contrast-induced nephropathy after primary percutaneous coronary intervention. Int Urol Nephrol 44:221-229

24. Mager A, Vaknin Assa H, Lev EI, Bental T, Assali A, Kornowski $\mathrm{R}$ (2011) The ratio of contrast volume to glomerular filtration rate predicts outcomes after percutaneous coronary intervention for STsegment elevation acute myocardial infarction. Catheter Cardiovasc Interv 78:198-201

25. Nozue T, Michishita I, Iwaki T, Mizuguchi I, Miura M (2009) Contrast medium volume to estimated glomerular filtration rate ratio as a predictor of contrast-induced nephropathy developing after elective percutaneous coronary intervention. J Cardiol 54:214-220

26. Yoon HJ, Hur SH (2011) Determination of safe contrast media dosage to estimated glomerular filtration rate ratios to avoid contrast-induced nephropathy after elective percutaneous coronary intervention. Korean Circ J 41:265-271

27. Tan N, Liu Y, Zhou YL, He PC, Yang JQ, Luo JF, Chen JY (2011) Contrast medium volume to creatinine clearance ratio: a predictor of contrast-induced nephropathy in the first 72 hours following percutaneous coronary intervention. Catheter Cardiovasc Interv 79:70-75

28. Polena S, Yang S, Alam R, Gricius J, Gupta JR, Badalova N, Chuang P, Gintautas J, Conetta R (2005) Nephropathy in critically Ill patients without preexisting renal disease. Proc West Pharmacol Soc 48:134-135

29. Lakhal K, Ehrmann S, Chaari A, Laissy JP, Regnier B, Wolff M, Pajot O (2011) Acute Kidney Injury Network definition of contrast-induced nephropathy in the critically ill: incidence and outcome. J Crit Care 26:593-599

30. Mitchell AM, Jones AE, Tumlin JA, Kline JA (2010) Incidence of contrast-induced nephropathy after contrast-enhanced computed tomography in the outpatient setting. Clin J Am Soc Nephrol 5:4-9
31. From AM, Bartholmai BJ, Williams AW, Cha SS, McDonald FS (2008) Mortality associated with nephropathy after radiographic contrast exposure. Mayo Clin Proc 83:1095-1100

32. Aspelin P, Almen T (1976) Studies on the acute toxicity of ionic and non-ionic contrast media following rapid intravenous injection. An experimental study in mice. Invest Radiol 11:309-314

33. Barrett BJ, Katzberg RW, Thomsen HS, Chen N, Sahani D, Soulez G, Heiken JP, Lepanto L, Ni ZH, Nelson R (2006) Contrastinduced nephropathy in patients with chronic kidney disease undergoing computed tomography: a double-blind comparison of iodixanol and iopamidol. Invest Radiol 41:815-821

34. Kuhn MJ, Chen N, Sahani DV, Reimer D, van Beek EJ, Heiken JP, So GJ (2008) The PREDICT study: a randomized double-blind comparison of contrast-induced nephropathy after low- or isoosmolar contrast agent exposure. AJR Am J Roentgenol 191:151-157

35. Thomsen HS, Morcos SK, Erley CM, Grazioli L, Bonomo L, Ni Z, Romano L (2008) The ACTIVE Trial: comparison on the effects on renal function of iomeprol-400 and iodixanol-320 in patients with chronic kidney disease undergoing abdominal computed tomography. Invest Radiol 43:170-178

36. Weisbord SD, Mor MK, Resnick AL, Hartwig KC, Palevsky PM, Fine MJ (2008) Incidence and outcomes of contrast-induced AKI following computed tomography. Clin J Am Soc Nephrol 3:1274-1281

37. Bruce RJ, Djamali A, Shinki K, Michel SJ, Fine JP, Pozniak MA (2009) Background fluctuation of kidney function versus contrastinduced nephrotoxicity. AJR Am J Roentgenol 192:711-718

38. Kakkar R, Sobieszczyk P, Binkert CA, Faxon DP, Mortele KJ, Singh AK (2008) Prevention of intravenous contrast-induced nephropathy in hospital inpatients. Crit Pathw Cardiol 7:1-4

39. Awai K, Hiraishi K, Hori S (2004) Effect of contrast material injection duration and rate on aortic peak time and peak enhancement at dynamic CT involving injection protocol with dose tailored to patient weight. Radiology 230:142-150

40. Awai K, Hori S (2003) Effect of contrast injection protocol with dose tailored to patient weight and fixed injection duration on aortic and hepatic enhancement at multidetector-row helical CT. Eur Radiol 13:2155-2160

41. Kristiansson M, Holmquist F, Nyman U (2010) Ultralow contrast medium doses at CT to diagnose pulmonary embolism in patients with moderate to severe renal impairment. A feasibility study. Eur Radiol 20:1321-1330

42. Nyman U, Elmstahl B, Geijer H, Leander P, Almen T, Nilsson M (2011) Iodine contrast iso-attenuating with diagnostic gadolinium doses in CTA and angiography results in ultra-low iodine doses. A way to avoid both CIN and NSF in azotemic patients? Eur Radiol $21: 326-336$

43. Lufft V, Hoogestraat-Lufft L, Fels LM, Egbeyong-Baiyee D, Tusch G, Galanski M, Olbricht CJ (2002) Contrast media nephropathy: intravenous CT angiography versus intraarterial digital subtraction angiography in renal artery stenosis: a prospective randomized trial. Am J Kidney Dis 40:236-242

44. Kolehmainen H, Sovia M (2003) Comparison of Xenetix 300 and Visipaque 320 in patients with renal failure (P27). 10th European Symposium on Urogenital Radiology. Eur Radiol 13:B32-B33

45. Garcia-Ruiz C, Martinez-Vea A, Sempere T, Sauri A, Olona M, Peralta C, Oliver A (2004) Low risk of contrast nephropathy in high-risk patients undergoing spiral computed tomography angiography with the contrast medium iopromide and prophylactic oral hydratation. Clin Nephrol 61:170-176

46. Becker CR, Reiser MF (2005) Use of iso-osmolar nonionic dimeric contrast media in multidetector row computed tomography angiography for patients with renal impairment. Invest Radiol 40:672-675

47. Kim SM, Cha RH, Lee JP, Kim DK, Oh KH, Joo KW, Lim CS, Kim S, Kim YS (2010) Incidence and outcomes of contrast-induced nephropathy after computed tomography in patients with CKD: a quality improvement report. Am J Kidney Dis 55:1018-1025 